\title{
Using Available Volume to Predict Fluid Diffusivity in Random Media
}

\author{
Jeetain Mittal,, , * Jeffrey R. Errington, ${ }^{2,+}$ and Thomas M. Truskett ${ }^{1,3,+}$ \\ ${ }^{1}$ Department of Chemical Engineering, \\ The University of Texas at Austin, Austin, TX \\ ${ }^{2}$ Department of Chemical and Biological Engineering, \\ University at Buffalo, The State University of New York, Buffalo, NY \\ ${ }^{3}$ Institute for Theoretical Chemistry, \\ The University of Texas at Austin, Austin, TX
}

(Dated: September 16, 2018)

\begin{abstract}
We propose a simple equation for predicting self-diffusivity of fluids embedded in random matrices of identical, but dynamically frozen, particles (i.e., quenched-annealed systems). The only nontrivial input is the volume available to mobile particles, which also can be predicted for two common matrix types that reflect equilibrium and non-equilibrium fluid structures. The proposed equation can account for the large differences in mobility exhibited by quenched-annealed systems with indistinguishable static pair correlations, illustrating the key role that available volume plays in transport.
\end{abstract}

PACS numbers: $66.10 . \mathrm{Cb}, 46.65 .+\mathrm{g}, 61.20 . \mathrm{Ja}$ 
Fluid transport in random porous media is central to a host of natural phenomena and technological applications, from the function of biological cells to the performance of materials for membrane separations and heterogeneous catalysis. Although it is now appreciated that kinetic processes in these systems are intimately connected to their microstructures (pore volume, surface area, connectivity, etc.) [1], determining the precise structure-property relationship for the transport property of interest remains a formidable challenge.

One promising line of inquiry has been the exploration of simple model systems for which both transport properties and structure are amenable to theoretical analysis. In the case of diffusivity, earlier work has focused primarily on single particle transport through random configurations of static obstacles [2]. At low obstacle densities, particles show anomalous, sub-diffusive motion over intermediate time and length scales but recover normal diffusive behavior in the long time limit. On the other hand, sub-diffusive motion is observed for all times at high obstacle densities. Similar behavior is predicted for transport of single ions in disordered matrices of quenched charges [3] .

Self-diffusivities of highly coupled fluids in random media pose additional challenges and are also of great interest. Very recently, insightful molecular dynamics simulation [4] and mode-coupling theory [5] studies have been presented for the hard-sphere (HS) fluid embedded in disordered matrices of obstacle spheres. This type of model, introduced originally by Madden and Glandt [6], is referred to as a quenched-annealed (QA) system. To the best of our knowledge, no simple theoretical relationship between self-diffusivity of mobile particles, densities of mobile and matrix particles, and matrix microstructure has been proposed for QA media. As a result, a general consensus on which physical parameters are most important for understanding mobility in these model porous materials is lacking.

In this Rapid Communication, we introduce one such approximate relationship that we motivate by using a physical argument for how the matrix reduces the volume available for diffusion of the mobile particles. We test its predictions against the numerical results of molecular dynamics simulations for HS QA systems with matrices that reflect equilibrium and non-equilibrium structures, which serve as idealizations for two different classes of physically realizable materials. We then demonstrate how the available volume in both types of matrices can be accurately predicted directly from equilibrium properties of the bulk HS fluid. Finally, we test our equation for diffusivity against simulation results of QA systems comprising Lennard-Jones (LJ) particles. For all models investigated, we find good agree- 
ment with simulations over a wide range of parameters, with significant overpredictions of diffusivity occuring only for high matrix densities where limited connectivity of the available volume (not accounted for in our approximate equation) also hinders transport.

The protocols that we use to generate static matrices represent two of the most commonly employed in studies of QA systems [4, 6, 7]. In the first, $N_{\mathrm{M}}$ particles of diameter $\sigma$ are initially equilibrated in a volume $V$ at temperature $T$. They are subsequently quenched (i.e., frozen in place) in an equilibrium configuration, and a "fluid" (F) of $N_{\mathrm{F}}$ identical particles is added, equilibrated, and studied. This method of matrix generation is referred to as QA-M because the matrix (M) itself reflects an equilibrium configuration of density $\rho_{\mathrm{M}}=N_{\mathrm{M}} \sigma^{3} / V$ for the bulk system. QA-M matrices serve as elementary models of amorphous solid materials prepared, e.g, by very rapidly cooling gel-forming suspensions of proteins or colloids [7].

The second matrix generation protocol we study is referred to as QA-FM because it involves first equilibrating $N_{\mathrm{F}}+N_{\mathrm{M}}$ identical particles of diameter $\sigma$ in a volume $V$ at temperature $T$. Then, $N_{\mathrm{M}}$ of the particles are randomly selected and quenched to create the solid matrix. The other $N_{\mathrm{F}}$ particles constitute the mobile "fluid" of density $\rho_{\mathrm{F}}=N_{\mathrm{F}} \sigma^{3} / V$. QA-FM matrices are idealized models for templated porous solids synthesized by depleting a high density material of one of its components by, e.g., dissolution, reaction, or desorption [7].

Our approach for predicting the fluid self-diffusivities of these systems is motivated by three basic observations about their behaviors. (i) The total pair correlation functions of HS QA-M and QA-FM configurations, averaged over mobile and matrix particles together at the same total density $\rho_{\mathrm{F}}+\rho_{\mathrm{M}}$, are indistinguishable [4]. (ii) Yet, their fluid self-diffusivities generally differ significantly, even when compared at the same ratio of matrix to mobile particles. This has previously been interpreted as evidence that the differences in the dynamics of QA media cannot be predicted based on static structural information alone [4]. (iii) However, there is a key static property that distinguishes individual QA systems: the fraction of volume available to the mobile particle centers in the matrix, $V_{0} / V$ [7]. As we demonstrate below, differences in this quantity can largely account for the wide range of self-diffusivities exhibited by QA materials that are otherwise "structurally similar".

For example, contrast the behavior of a bulk HS fluid with density $\rho=\rho_{\mathrm{F}}+\rho_{\mathrm{M}}$ to a HS QA system (produced by either -M or -FM protocols) with matrix and mobile particle densities of $\rho_{\mathrm{M}}$ and $\rho_{\mathrm{F}}$, respectively. The binary collisions that mediate diffusion in these two systems are expected to be comparable, since their pair correlation functions and mobile- 
particle thermal velocities are the same. Nonetheless, mobile particles in the QA system will diffuse over a shorter characteristic length scale per unit time compared to those of the bulk because the volume available for diffusion in the QA matrix, $V_{0}$, is smaller than $V$. If we assume that the characteristic length scales for diffusion are proportional to the cube root of the respective available volumes and, in turn, that the associated self-diffusivities are proportional to the square of the length scales, we arrive at the following approximate relationship:

$$
D\left(\rho_{\mathrm{F}}, \rho_{\mathrm{M}}\right) \approx D\left(\rho_{\mathrm{F}}+\rho_{\mathrm{M}}, 0\right) \times\left(V_{0} / V\right)^{2 / 3},
$$

where $D\left(\rho_{\mathrm{F}}, \rho_{\mathrm{M}}\right)$ and $D\left(\rho_{\mathrm{F}}+\rho_{\mathrm{M}}, 0\right)$ are the self-diffusivities of the mobile particles in QA and bulk fluid systems. As we show below, the appeal of this relation is its ability to predict the self-diffusivity of non-trivial QA systems from knowledge of well-characterized equilibrium properties of the bulk HS fluid.

In HS QA-M systems, matrices are drawn from equilibrium configurations of the bulk HS fluid with density $\rho_{\mathrm{M}}$, and thus $V_{0} / V$ is given by [8]

$$
V_{0} / V=\exp \left[-\mu^{\mathrm{ex}}\left(\rho_{\mathrm{M}}\right) / k_{\mathrm{B}} T\right]
$$

The excess chemical potential (relative to ideal gas) of the HS fluid, $\mu^{\mathrm{ex}}$, can be obtained analytically from, e.g., either scaled particle theory [8] or the Carnahan-Starling (CS) equation of state 9] (here, we adopt the latter, which is very accurate for $\rho_{\mathrm{M}}<0.9$ ).

In HS QA-FM media, however, the matrix structure is different than that of an equilibrium HS fluid because it is quenched in the presence of mobile particles. Nonetheless, as Van Tassel and co-workers [7, 10] have demonstrated, because of the specific protocol by which "non-equilibrium" QA-FM matrices are created, one can still apply equilibrium liquid-state approaches (e.g., integral equation theories) to estimate $V_{0} / V$. Here, we introduce an accurate information-theory (IT) based stategy for accomplishing this task. As we show, its main advantage is that it only requires knowledge of $\rho_{\mathrm{M}}, \rho_{\mathrm{F}}$, and the pair correlation function $g(r)$ of the bulk HS fluid.

Our strategy implicitly uses the fact that the pair correlations of the HS QA-FM system are indistinguishable from those of an equilibrium HS fluid with density $\rho_{\mathrm{F}}+\rho_{\mathrm{M}}$. Since the QA-FM particle identities (matrix or mobile) are randomly distributed, one can write down an exact expression for $V_{0} / V$ in the QA-FM matrix in terms of equilibrium properties of 
bulk HS fluid:

$$
V_{0} / V=\sum_{i=0}^{\infty} \Pi_{i}\left(\rho_{\mathrm{M}}+\rho_{\mathrm{F}}\right) \times\left[1+\frac{\rho_{\mathrm{M}}}{\rho_{\mathrm{F}}}\right]^{-i} .
$$

Here $\Pi_{i}\left(\rho_{\mathrm{M}}+\rho_{\mathrm{F}}\right)$ represents the probability that a randomly placed spherical window (with radius equal to one particle diameter) in a bulk HS fluid of density $\rho_{\mathrm{F}}+\rho_{\mathrm{M}}$ will contain precisely $i$ particle centers (see Fig. (1). The quantitiy $\left[1+\rho_{\mathrm{M}} / \rho_{\mathrm{F}}\right]^{-i}$ is the probability that, in the structurally equivalent QA-FM system, all of the $i$ centers in the window would be fluid particles.

IT provides expressions for the $\Pi_{i}$,

$$
\Pi_{i}=\exp \left[\lambda_{0}+\lambda_{1} i+\lambda_{2} i^{2}\right] / i !
$$

which maximize a relative information entropy subject to some experimental constraints [11]. In particluar, $\lambda_{0}, \lambda_{1}$, and $\lambda_{2}$ are Lagrange multipliers determined (see, e.g., 11]) by imposing the normalization condition $\sum_{i} \Pi_{i}=1$ and the first two moments of the window occupancy, $\bar{i}$ and $\overline{i^{2}}$,

$$
\begin{aligned}
\bar{i} & =4 \pi \rho / 3 \\
\overline{i^{2}} & =\bar{i}+\rho^{2} \int_{\mathrm{w}} d \mathbf{r} \int_{\mathbf{w}} d \mathbf{r}^{\prime} g\left(\left|\mathbf{r}-\mathbf{r}^{\prime}\right|\right) .
\end{aligned}
$$

Here, $\rho=N \sigma^{3} / V$, and the subscript $\mathrm{w}$ in the last expression indicates that the integrals are constrained to the spherical observation window. Fig. 2 shows a numerical comparison of $V_{0} / V$ predicted by Eq. 3 and 4 to the "exact" results for $V_{0} / V$ obtained from applying the available space algorithm of Sastry et al. 12] to HS QA-FM matrices. As is evident, the simple IT approach provides accurate predictions over a wide range of matrix parameters.

Having established means for estimating $V_{0} / V$ of both QA-M and QA-FM matrices, we now only require an expression for the self-diffusivity of the bulk HS fluid, $D(\rho, 0)$, in order to predict HS QA diffusivity using Eq. 1. For this, we adopt Speedy's empirical fit to molecular dynamics simulation data [13],

$$
D(\rho, 0)=\frac{A}{\rho}\left(1-\frac{\rho}{1.09}\right)\left[1+\rho^{2}\left(0.4-0.83 \rho^{2}\right)\right],
$$

where $A=3 /(8 \sqrt{\pi})$. We implicitly non-dimensionalized $D(\rho, 0)$ of Eq. 66 and all other selfdiffusivities in this study, by $\sigma^{2} / \tau$, where $\tau=\sigma \sqrt{m / k_{\mathrm{B}} T}, k_{\mathrm{B}}$ is the Boltzmann's constant, and $m$ is particle mass. 
To test the validity of Eq. 1] we performed molecular dynamics simulations [14] for HS QA-M and QA-FM systems in the microcanonical ensemble using $N_{\mathrm{F}}=1500$ mobile particles and a periodically-replicated simulation cell. Runs were performed at different values of $N_{\mathrm{M}}$ and $V / \sigma^{3}$ to obtain results for specific combinations of $\rho_{\mathrm{F}}$ and $\rho_{\mathrm{M}}$. We extracted selfdiffusivity of the mobile particles $D\left(\rho_{\mathrm{F}}, \rho_{\mathrm{M}}\right)$ by fitting the long-time $(t \gg 1)$ behavior of the average mean-squared displacement to the Einstein relation $\left\langle\Delta \mathbf{r}^{2}\right\rangle=6 D t$.

Fig. 3] provides a fairly comprehensive comparison of $D\left(\rho_{\mathrm{F}}, \rho_{\mathrm{M}}\right)$ from Eq. 1] to the results of our molecular dynamics simulations. Interestingly, the predictions show semi-quantitative agreement with simulations of QA systems for matrix densities in the wide range $0 \leq \rho_{\mathrm{M}} \leq$ 0.25, which includes systems where matrix particles exclude mobile particle centers from over $70 \%$ of the total volume (see, e.g., Fig. 2). Given that matrix particles reduce $D\left(\rho_{\mathrm{F}}, \rho_{\mathrm{M}}\right)$ by more than an order of magnitude across this range of densities, the success of Eq. 1 argues that available volume plays a primary role in controlling the single-particle dynamics. For matrix densities greater than $\rho_{\mathrm{M}}=0.25$ (i.e., systems with less than $30 \%$ of volume available to mobile particle centers), Eq. 11 captures the qualitative trends, but it systematically overpredicts the simulated self-diffusivities. This overprediction is, of course, expected, given that Eq. 1 only accounts for the reduction of available volume and not the fact that available volume also becomes highly disconnected (i.e., some pockets of available volume are inaccessible or are accessible by only small number of paths) at high matrix densities [1], which acts to further hinder transport. However, for systems of physical relevance (porous catalysts, membranes for separations, transport gels for drug delivery, etc.) which rely on rapid diffusion for their functionality, the precise connectivity of the available volume will be less important.

In order to test whether the connection between diffusivity and available volume holds more generally for fluids confined to random media, we also performed molecular dynamics simulations of QA-FM systems comprising LJ particles (truncated and shifted with a quadratic function in $r$ [15] to insure that both the potential and its gradient vanish at $\left.r_{\text {cut }}=2.5\right)$. The details of the simulations are identical to those of the HS QA systems described earlier, except that the equations of motion were integrated via the velocity Verlet algorithm [14], and $N_{\mathrm{F}}=1000$ mobile particles were considered. The goal was to test if one could employ techniques commonly used in thermodynamic perturbation theory to map the QA LJ system onto an equivalent QA HS system, and then use Eq. 11 to predict 
the self-diffusivity. The specific mapping that we used in this study is a Boltzmann factor criterion [16, 17], which determines the temperature-dependent "effective" HS diameter $\sigma(T)$ (and hence the corresponding reduced matrix and fluid denisties, $\rho_{\mathrm{M}}$ and $\rho_{\mathrm{F}}$ ) of the LJ system through the following relationship $u_{0}(r=\sigma)=k_{\mathrm{B}} T$, where $u_{0}$ is the repulsive part of the Weeks-Chandler-Andersen decomposition of the pair potential [18]. The hypothesis is that LJ QA systems will exhibit similar reduced diffusivities $D\left(\rho_{\mathrm{F}}, \rho_{\mathrm{M}}\right)$ as HS QA systems when compared at the same values of $\rho_{\mathrm{F}}$ and $\rho_{\mathrm{M}}$.

As a first test of this idea, we compare in Fig. 4a the diffusivities $D\left(\rho_{\mathrm{F}}, \rho_{\mathrm{M}}\right)$ of the HS QA-FM and LJ QA-FM systems obtained by our molecular dynamics simulations. In this plot, the LJ QA-FM system is at a temperature $T=\epsilon / k_{\mathrm{B}}$, where $\epsilon$ is the characteristic energy of the LJ pair potential [15]. Except for at very low fluid densities (conditions for which the LJ fluid structure is not accurately approximated by an equivalent HS reference fluid [19]), the mapping brings the dynamics of the LJ and HS QA systems into excellent agreement.

A final test is to check whether the simple aforementioned mapping allows direct prediction of LJ QA-FM dynamics at other temperatures using Eq. 1. Fig. 4b shows the comparison of the predicted $D\left(\rho_{\mathrm{F}}, \rho_{\mathrm{M}}\right)$ versus the results of our molecular dynamics simulations at $T=3 \epsilon / k_{\mathrm{B}}$. The agreement between the predictions and the simulations again confirm the pivotal role that available volume plays in controlling the single-particle dynamics of fluids in porous media.

TMT and JRE acknowledge the financial support of the National Science Foundation Grants No. CTS-0448721 and CTS-028772, respectively, and the Donors of the American Chemical Society Petroleum Research Fund Grants No. 41432-G5 and 43452-AC5, respectively. TMT also acknowledges the support of the David and Lucile Packard and Alfred P. Sloan Foundations. The Texas Advanced Computing Center (TACC) provided computational resources for this study.

* jeetain@che.utexas.edu

† jerring@buffalo.edu

‡ truskett@che.utexas.edu 
[1] S. Torquato, Random Heterogeneous Materials: Microstructure and Manroscopic Properties (Springer-Verlag, New York, 2002).

[2] A. Bunde and S. Havlin, Fractals and Disordered Systems (Springer, Berlin, 1991).

[3] M. W. Deem and D. Chandler, J. Stat. Phys. 76, 911 (1994).

[4] R. Chang, K. Jagannathan, and A. Yethiraj, Phys. Rev. E 69, 051101/1 (2004).

[5] V. Krakoviack, Phys. Rev. Lett. 94, 065703 (2005).

[6] W. G. Madden and E. D. Glandt, J. Stat. Phys. 51, 537 (1988).

[7] P. R. Van Tassel, J. Talbot, P. Viot, and G. Tarjus, Phys. Rev. E 56, R1299 (1997).

[8] H. Reiss, H. L. Frisch, and J. L. Lebowitz, J. Chem. Phys. 31, 369 (1959).

[9] N. F. Carnahan and K. E. Starling, J. Chem. Phys. 51, 635 (1969).

[10] P. R. Van Tassel, J. Chem. Phys. 107, 9530 (1997).

[11] G. Hummer, S. Garde, A. E. Garcia, M. E. Paulaitis, and L. R. Pratt, J. Phys. Chem. B 51, 10469 (1998).

[12] S. Sastry, D. S. Corti, P. G. Debenedetti, and F. H. Stillinger, Phys. Rev. E 56, 5524 (1997).

[13] R. J. Speedy, Mol. Phys. 62, 509 (1987).

[14] D. C. Rapaport, The Art of Molecular Dynamics Simulation (Cambridge University Press, 2004), 2nd ed.

[15] S. D. Stoddard and J. Ford, Phys. Rev. A 8, 1504 (1973).

[16] C. S. Hsu, D. Chandler, and L. J. Loweden, J. Chem. Phys. 14, 213 (1976).

[17] D. Ben-Amotz and G. Stell, J. Phys. Chem. B 108, 6877 (2004).

[18] D. Chandler, J. D. Weeks, and H. C. Andersen, Science 220, 787 (1983).

[19] J. R. Errington, P. G. Debenedetti, and S. Torquato, J. Chem. Phys. 118, 2256 (2003). 


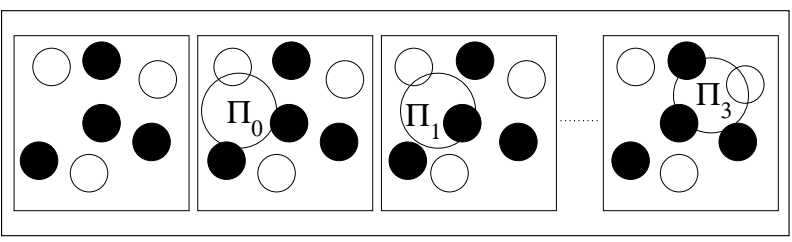

FIG. 1: Schematic of a QA system. Equi-sized filled and empty circles are matrix and fluid particles, respectively. The bigger circle is a window with radius equal to the one particle diameter. $\Pi_{i}$ is the probability that it will be occupied by exactly $i$ matrix or fluid particle centers. 


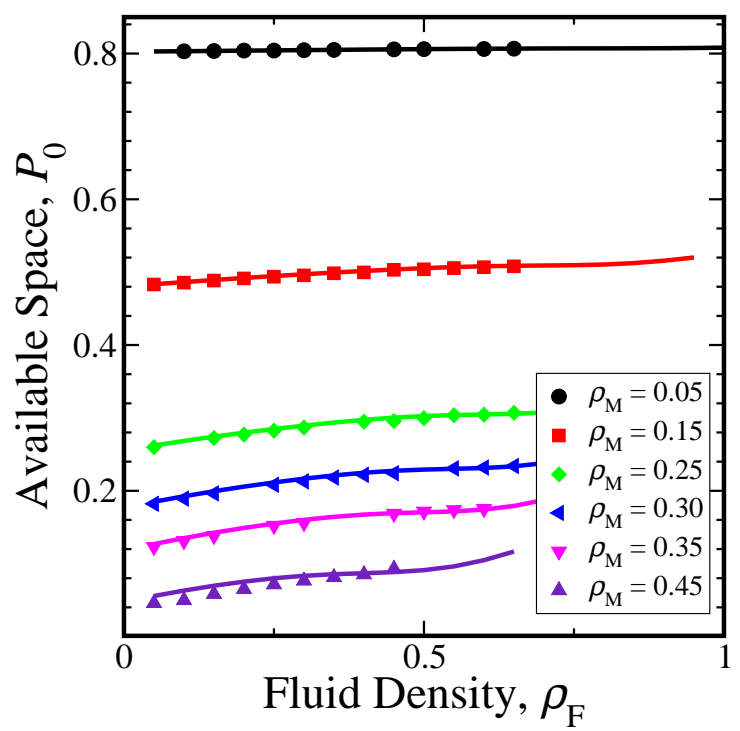

FIG. 2: The fractional available volume $V_{0} / V$ in HS QA-FM matrices computed using the IT approach of Eq. 3 and 4 (curves) and "exact" results (filled circles) using the available space algorithm of Ref [12]. 


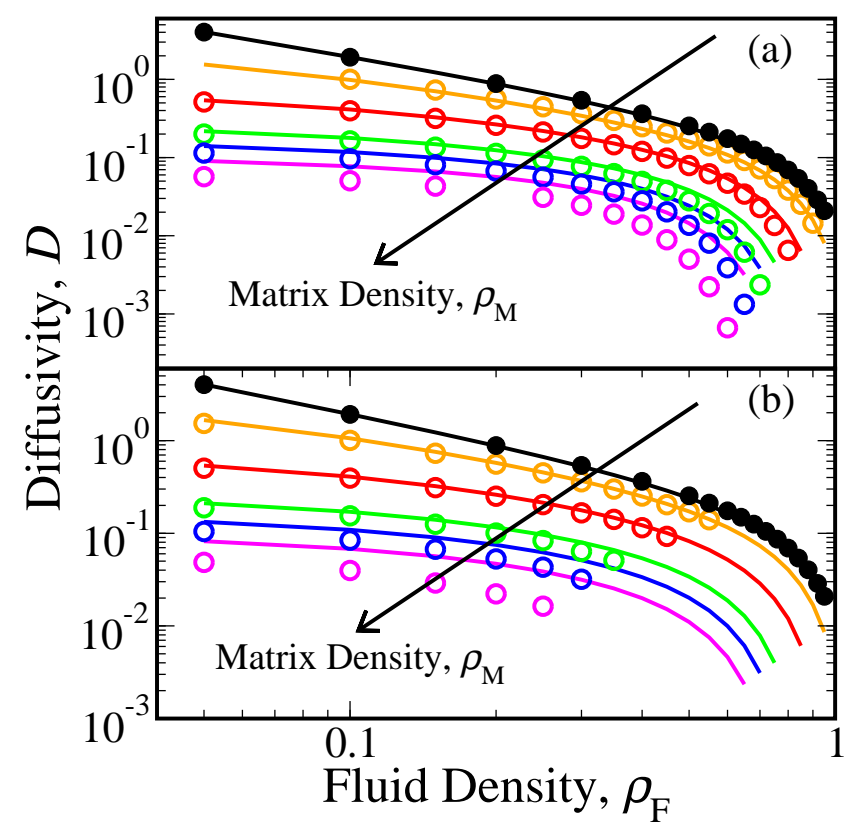

FIG. 3: Fluid self-diffusivity $D$ versus fluid density $\rho_{\mathrm{F}}$ for (a) HS QA-FM and (b) HS QA-M systems with matrix densities $\rho_{\mathrm{M}}=0.0,0.05,0.15,0.25,0.30$, and 0.35 . Curves are predictions of Eq. 1] and circles are molecular dynamics simulation results. 


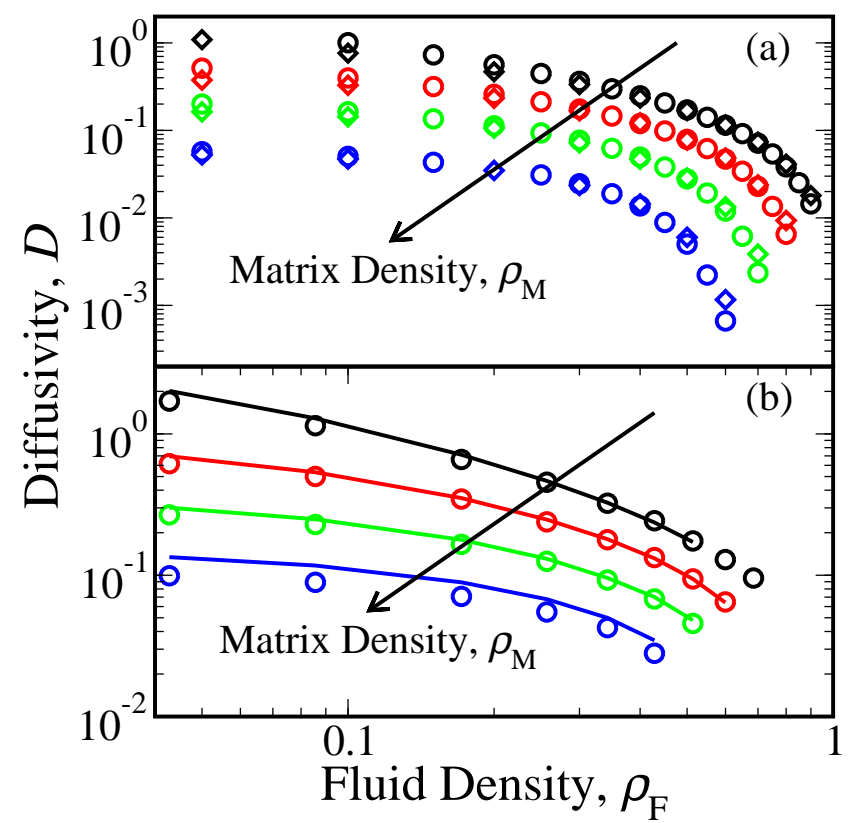

FIG. 4: (a) Fluid self-diffusivity $D$ versus fluid density $\rho_{\mathrm{F}}$ for HS QA-FM (circles) and LJ QAFM (diamonds) systems obtained via molecular dynamics simulations. The LJ QA-FM system is at $T=\epsilon / k_{\mathrm{B}}$. Matrix densities of $\rho_{\mathrm{M}}=0.05,0.15,0.25$, and 0.35 are presented. (b) Results for the LJ QA-FM system at $T=3 \epsilon / k_{\mathrm{B}}$ : molecular dynamics simulations (circles) and Eq. 1] (curves). Matrix densities of $\rho_{\mathrm{M}}=0.043,0.128,0.214$, and 0.3 are presented. In both (a) and (b), matrix and mobile densities for the LJ QA-FM systems are defined as $\rho_{\mathrm{M}}=N_{\mathrm{M}} \sigma(T)^{3} / V$ and $\rho_{\mathrm{F}}=N_{\mathrm{F}} \sigma(T)^{3} / V$, respectively. The effective HS diameter $\sigma(T)$ is determined by a Boltzmann factor criterion described in the text. 